\title{
The biennial life strategy in a random environment
} Supplement

\section{J. B. T. M. Roerdink}

Centre for Mathematics and Computer Science, Kruislaan 413, 1098 SJ Amsterdam, The Netherlands

\begin{abstract}
In a previous paper (J. Math. Biol. 26, 199-215 (1988)) we calculated the mean and variance of the long-run geometric growth rate of a discrete-time population model with two age classes in a random environment. The formula which was used in that paper as the starting point for the computation of the variance represents only the contribution of the one-period variances. Here we supplement these results by a calculation of the exact variance. All qualitative conclusions reached before are unaffected.
\end{abstract}

Key words: Population biology - Random matrix products - Variance of the geometric growth rate

\section{Introduction}

In a previous paper [1], referred to as $I$ in the following, we studied the mean and variance of the geometric growth rate for the following discrete-time model of a biennial plant population:

$$
n_{t+1}=X_{t+1} n_{t}, \quad t=0,1,2, \ldots
$$

where $X_{t}$ is the nonnegative matrix

$$
X_{t}=\left[\begin{array}{cc}
0 & f \phi_{t} \\
s & (1-f) s
\end{array}\right] .
$$

Here $t$ is a time just before flowering, $n_{t}$ is the population vector $n_{t}=\left(N_{1, t}, N_{2, t}\right)^{\prime}$ and

$N_{1, t}$ the number of individuals which are one year old at time $t$;

$N_{2, t}$ the number of individuals older than one year at time $t$;

$s$ survival probability per year of individuals older than one year, $0<s<1$; $f$ fraction of individuals older than one year that flowers in a given year, $0 \leqslant f \leqslant 1$

$\phi_{t}$ number of offspring per flowering plant after one year, $\phi_{t} \geqslant 0$.

We assumed $\left\{\phi_{t}\right\}$ to be an independent identically-distributed sequence of random 
variables with common density

$$
g(\phi)=\left\{k^{a} / \Gamma(a)\right\} \phi^{a-1} e^{-k \phi}, \quad a>0, \quad k>0,
$$

where $\Gamma(a)$ is the gamma function.

In order to define the (scaled) moments of the geometric growth rate we introduce the following notation. For each vector $n$ with nonnegative components $n_{i}, i=1,2, \ldots, d$, we denote by $|n|=n_{1}+n_{2}+\cdots \dot{n}_{d}$ the norm of the vector $n$ (the total population), and by $\bar{n}=n /|n|$ the unit vector in the direction of $n$ (the age structure). The space of age structures is the simplex

$$
C=\left\{n \in \mathbb{R}^{d}: n_{i} \geqslant 0, n_{1}+n_{2}+\cdots n_{d}=1\right\} .
$$

From the difference equation (1.1a) one obtains

$$
\ln \left(\frac{\left|n_{t}\right|}{\left|n_{0}\right|}\right)=\sum_{i=1}^{t} r_{i}
$$

where $r_{i}$ is the one-period growth rate at time $i$,

$$
r_{i}=\ln \left(\frac{\left|n_{i}\right|}{\left|n_{i-1}\right|}\right)=\ln \left(\left|X_{i} \bar{n}_{i-1}\right|\right) .
$$

The mean geometric growth rate is defined as

$$
y:=\lim _{t \rightarrow \infty} \frac{1}{t} \mathbb{E}\left\{\ln \left(\frac{\left|n_{t}\right|}{\left|n_{0}\right|}\right)\right\},
$$

where $\mathbb{E}$ denotes mathematical expectation over the distribution of the random matrices. Using (1.4) and the i.i.d. property of the sequence $\left\{X_{i}\right\}$ one finds the general formula for the mean of the long-run geometric growth rate which we used in $I$,

$$
\gamma=\iint \ln |X \bar{n}| d \mu(X) d \nu(\bar{n}) .
$$

Here $\mu$ is the common distribution of the matrices $\left\{X_{t}\right\}$ and $\nu$ the unique $\mu$-invariant measure (the stationary age-structure distribution) on the simplex $C$.

For the variance $\sigma^{2}$ of the geometric growth rate we find from (1.4), denoting by $\operatorname{Var}\{\cdots\}(\operatorname{Covar}\{\cdots, \cdots\})$ the variance (covariance) of the bracketed expression,

$$
\sigma^{2}:=\lim _{t \rightarrow \infty} \frac{1}{t} \operatorname{Var}\left\{\ln \left(\frac{\left|n_{t}\right|}{\left|n_{0}\right|}\right)\right\}=\operatorname{VAR}+\text { COVAR, }
$$

where VAR is the contribution of the one-period variances,

$$
\operatorname{VAR}=\lim _{t \rightarrow \infty} \frac{1}{t} \sum_{i=1}^{t} \operatorname{Var}\left\{r_{i}\right\}=-\gamma^{2}+\iint\{\ln |X \bar{n}|\}^{2} d \mu(X) d \nu(\bar{n}),
$$

and COVAR the contribution of the inter-period covariances,

$$
\operatorname{COVAR}=\lim _{t \rightarrow \infty} \frac{1}{t} \sum_{i \neq j}^{t} \operatorname{Covar}\left\{r_{i}, r_{j}\right\}
$$

The formula (3.9b) of I which we used for the variance $\sigma^{2}$ of the geometric growth rate coincides with (1.8) and therefore incorporates only the contribution 
of the one-period variances ${ }^{1}$. In this paper we will discuss a method to calculate the exact variance $\sigma^{2}$ of the geometric growth rate. We reduce the computation of $\sigma^{2}$ to the solution of a linear integral equation followed by quadrature. This is carried out numerically for the parameter values used in I. We compare the results with the previous ones based upon the expression (1.8). The conclusions are: (i) all results of I remain qualitatively correct; (ii) the small noise and lognormal approximations of $\sigma^{2}$ are seen to be better than the results of I suggested, although they remain inaccurate for values of the flowering fraction near unity.

The organization of the paper is as follows. In Sect. 2 we present a general method to compute the variance of the geometric growth rate, starting from the work of Le Page on the central limit theorem for products of i.i.d. random matrices, see [2]. This method is applied to the model (1.1) in Sect. 3. Section 4 contains the results and a discussion of the numerical calculations.

\section{The exact variance (general case)}

Our method to compute the exact variance of the geometric growth rate is based upon the following results of Le Page as described in [2], part A, chap. V, in particular Theorem 4.3 and Lemma 5.2; the corresponding irreducibility and moment conditions are satisfied in our case for $f \in(0,1)$, with $f$ the flowering fraction. First define the Fourier-Laplace operator $T(z), z \in \mathbb{C}$, acting on a space of Hölder-continuous functions $f(\bar{n})$ on the simplex $C$, by

$$
[T(z) f](\bar{n})=\int e^{z \ln |X \bar{n}|} f(X \cdot \bar{n}) d \mu(X)
$$

where

$$
X \cdot \bar{n}:=X n /|X n| .
$$

Then (see [2] for details):

(i) the operator $T(z)$ has a unique eigenvalue $\lambda(z)$ of maximum modulus;

(ii) $T$ and $\lambda$ are analytic functions of $z$ near the origin;

$$
\begin{aligned}
\lambda(0) & =1, \\
\lambda^{\prime}(0) & =\gamma, \\
\lambda^{\prime \prime}(0) & =\sigma^{2}+\gamma^{2},
\end{aligned}
$$

primes denoting differentiation with respect to $z$.

Therefore the mean $\gamma$ and variance $\sigma^{2}$ of the geometric growth rate can be expressed in terms of the derivatives of $\lambda$ with respect to $z$.

After these prerequisites we proceed to an explicit determination of the derivatives in (2.3). Let the eigenfunction belonging to the eigenvalue $\lambda(z)$ be denoted by $f(\bar{n}, z)$, so that

$$
[T(z) f](\bar{n}, z)=\lambda(z) f(\bar{n}, z) .
$$

\footnotetext{
1 This was pointed out by S. Orzack. We are indebted to S. D. Tuljapurkar for bringing this to our attention
} 
We assume the normalization condition

$$
\int f(\bar{n}, z) d \nu(\bar{n})=1
$$

where $\nu(\bar{n})$ is the invariant measure on the space $C$ of age structures. Because of the analyticity property we can expand $T(z), \lambda(z)$ and $f(\bar{n}, z)$ in power series in $z$ :

$$
\begin{aligned}
T(z) & =T(0)+z T^{\prime}(0)+\frac{1}{2} z^{2} T^{\prime \prime}(0)+\cdots, \\
\lambda(z) & =\lambda(0)+z \lambda^{\prime}(0)+\frac{1}{2} z^{2} \lambda^{\prime \prime}(0)+\cdots, \\
f(\bar{n}, z) & =f_{0}(\bar{n})+z f_{1}(\bar{n})+\frac{1}{2} z^{2} f_{2}(\bar{n})+\cdots,
\end{aligned}
$$

where from (2.5),

$$
\begin{aligned}
& \int f_{0}(\bar{n}) d \nu(\bar{n})=1, \\
& \int f_{i}(\bar{n}) d \nu(\bar{n})=0, \quad i=1,2, \ldots
\end{aligned}
$$

Now we insert the expansions (2.6) in (2.4) and collect terms of equal orders in $z$.

(i) Terms of order zero

Collecting all terms which are independent of $z$ we find

$$
\int f_{0}(X \cdot \bar{n}) d \mu(X)=\lambda(0) f_{0}(\bar{n})
$$

Next we multiply (2.8) by $d \nu$ and integrate. At this point we notice that the $\mu$-invariance of the measure $\nu$ is expressed through the equation

$$
\iint F(X \cdot \bar{n}) d \mu(X) d \nu(\bar{n})=\int F(\bar{n}) d \nu(\bar{n}),
$$

valid for any bounded Borel function $F$. Using this identity and the normalization condition (2.7a) one finds $\lambda(0)=1$, in accordance with $(2.3 \mathrm{a})$. Therefore $f_{0}(\tilde{n})=$ constant is a solution and, again taking into account $(2.7 \mathrm{a})$, we have

$$
f_{0}(\bar{n})=1 \text {. }
$$

\section{(ii) Terms of order one}

The next order in $z$ yields

$$
\int \ln |X \bar{n}| f_{0}(X \cdot \bar{n}) d \mu(X)+\int f_{1}(X \cdot \bar{n}) d \mu(X)=\lambda^{\prime}(0) f_{0}(\bar{n})+\lambda(0) f_{1}(\bar{n}) .
$$

Again integrating this equation over $d \nu$ and using (2.7), (2.9) and (2.10) one finds

$$
\lambda^{\prime}(0)=\iint \ln |X \bar{n}| d \mu(X) d \nu(\bar{n}),
$$


in agreement with (2.3b) and (1.5). Using (2.3a), (2.10) and (2.12) in (2.11) we find the following integral equation for $f_{1}$,

$$
f_{1}(\bar{n})=\int f_{1}(X \cdot \bar{n}) d \mu(X)+\varepsilon(\bar{n})
$$

where

$$
\varepsilon(\bar{n})=\int \ln |X \bar{n}| d \mu(X)-\gamma
$$

with (see (1.5)),

$$
\int \varepsilon(\bar{n}) d \nu(\bar{n})=0
$$

(iii) Terms of order two

Finally we collect all terms of second order in $z$ to find

$$
\begin{gathered}
\int\left[\frac{1}{2}\{\ln |X \bar{n}|\}^{2} f_{0}(X \cdot \bar{n})+\ln |X \bar{n}| f_{1}(X \cdot \bar{n})+\frac{1}{2} f_{2}(X \cdot \bar{n})\right] d \mu(X) \\
=\frac{1}{2} \lambda^{\prime \prime}(0) f_{0}(\bar{n})+\lambda^{\prime}(0) f_{1}(\bar{n})+\frac{1}{2} \lambda(0) f_{2}(\bar{n}) .
\end{gathered}
$$

Once more we integrate over $d \nu$ and use (2.7), (2.9) and (2.10) to arrive at

$\lambda^{\prime \prime}(0)=\iint\{\ln |X \bar{n}|\}^{2} d \mu(X) d \nu(\bar{n})+2 \iint \ln |X \bar{n}| f_{1}(X \cdot \bar{n}) d \mu(X) d \nu(\bar{n})$.

Writing the exact variance $\sigma^{2}$ of the geometric growth rate in the form (1.7),

$$
\sigma^{2}=\mathrm{VAR}+\mathrm{COVAR}
$$

where VAR is identical to (1.8), we therefore find from (2.3c) and (2.15) the following expression for the term COVAR as defined in (1.9),

$$
\text { COVAR }=2 \iint \ln |X \bar{n}| f_{1}(X \cdot \bar{n}) d \mu(X) d \nu(\bar{n}) .
$$

In the next section we specialize these results to our population model (1.1).

\section{The exact model variance}

First we recapitulate some results from I. Instead of age-structure $\bar{n}$ we consider the ratio

$$
r_{t}=N_{2, t} / N_{1, t}
$$

as well as a scaled version of this,

$$
\tau_{t}=r_{t} /(1-f),
$$

where $f$ is the flowering fraction. From (1.1) we have the recursions

$$
r_{t+1}=f \phi_{t} /\left\{s r_{t}+s(1-f)\right\}, \quad \tau_{t+1}=\eta \phi_{t} /\left(1+\tau_{t}\right),
$$


where

$$
\eta=f\left\{(1-f)^{2} s\right\}^{-1} .
$$

We write the invariant measure in terms of the variable $\tau$ and denote it by $\nu(\tau)$. Its density $h(\tau)$ satisfies the integral equation

$$
h(\tau)=\int_{0}^{\infty} h\left(\tau^{\prime}\right) g\left(\frac{\left(\tau^{\prime}+1\right) \tau}{\eta}\right)\left\{\frac{\tau^{\prime}+1}{\eta}\right\} d \tau^{\prime},
$$

where $g(\cdot)$ is the density (1.2) of the gamma distribution. The solution of (3.5) is

$$
h(\tau)=K^{-1} \tau^{a-1}(1+\tau)^{-a} e^{-z \tau}, \quad K=\int_{0}^{\infty} \tau^{a-1}(1+\tau)^{-a} e^{-z \tau},
$$

with $z$ (unrelated to the parameter $z$ in the previous section) defined by

$$
z=k / \eta=k s(1-f)^{2} / f \text {. }
$$

For later use we write Eq. (3.5) in the form

$$
h(\tau)=\int_{0}^{\infty} K\left(\tau, \tau^{\prime}\right) h\left(\tau^{\prime}\right) d \tau^{\prime}
$$

where the kernel $K(\cdot, \cdot)$ is given by

$$
K\left(\tau, \tau^{\prime}\right)=\left\{z^{a} / \Gamma(a)\right\} \tau^{a-1}\left(1+\tau^{\prime}\right)^{a} e^{-z \tau\left(1+\tau^{\prime}\right)},
$$

with

$$
\int_{0}^{\infty} K\left(\tau, \tau^{\prime}\right) d \tau=1
$$

and the symmetry relation

$$
K\left(\tau, \tau^{\prime}\right) h\left(\tau^{\prime}\right)=K\left(\tau^{\prime}, \tau\right) h(\tau)
$$

The average geometric growth rate $\gamma$ is finally given by

$$
\gamma=\int_{0}^{\infty}[\ln \{s(1-f)(1+\tau)\}] h(\tau) d \tau .
$$

Having recalled these results from I we next look at the integral equation (2.13a) for $f_{1}$. In this equation we go over to the new variable $\tau$ as well and write $f_{1}(\tau)$ instead of $f_{1}(\bar{n})$, etc. Since

$$
|X \bar{n}|=\frac{f \phi+s r+s(1-f)}{1+r}=\frac{f \phi+s(1-f)(1+\tau)}{1+(1-f) \tau},
$$

where $r$ and $\tau$ are defined as in (3.1) and (3.2) with subscripts omitted, we find from (2.13),

$$
f_{1}(\tau)=\int_{0}^{\infty} f_{1}\left(\frac{\eta \phi}{1+\tau}\right) g(\phi) d \phi+\varepsilon(\tau)
$$

where

$$
\varepsilon(\tau)=\int_{0}^{\infty} \ln \left\{\frac{f \phi+s(1-f)(1+\tau)}{1+(1-f) \tau}\right\} g(\phi) d \phi-\gamma
$$


Making a change of variables, $\tau^{\prime}=\eta \phi /(1+\tau)$, in (3.12) we find

$$
f_{1}(\tau)=\int_{0}^{\infty} K\left(\tau^{\prime}, \tau\right) f_{1}\left(\tau^{\prime}\right) d \tau^{\prime}+\varepsilon(\tau) .
$$

Notice that the kernel of this integral equation is the adjoint of that occurring in (3.8). Because of (3.9b) the solution of the homogeneous equation corresponding to $(3.14 \mathrm{a})$ is $f_{1}(\tau)=$ constant. Therefore (3.14a) has infinitely many solutions. We get a unique solution by imposing the normalization condition $(2.7 \mathrm{~b})$,

$$
\int_{0}^{\infty} f_{1}(\tau) h(\tau) d \tau=0 .
$$

To obtain the contribution COVAR to the variance $\sigma^{2}$ of the geometric growth rate one has to solve the integral equation (3.14) for $f_{1}(\tau)$, substitute the result in (2.17) and carry out the integrations. It turns out that in fact we can reduce the double integration to a single one by combining the contributions VAR and COVAR. To achieve this we introduce the auxiliary functions $\rho(\tau)$ and $\xi(\tau)$ by

$$
\begin{gathered}
\rho(\tau)=f_{1}(\tau)+\ln [1+(1-f) \tau], \\
\xi(\tau)=\ln \{s(1-f)(1+\tau)\}-\gamma .
\end{gathered}
$$

Then after a considerable number of algebraic manipulations which are outlined in Appendix A, we arrive at the following end result for the variance:

$$
\sigma^{2}=\int_{0}^{\infty} \xi(\tau)\{2 \rho(\tau)-\xi(\tau)\} h(\tau) d \tau,
$$

where $\rho$ obeys the Fredholm integral equation of the second kind

$$
\rho(\tau)=\int_{0}^{\infty}\left\{K\left(\tau^{\prime}, \tau\right)-h\left(\tau^{\prime}\right)\right\} \rho\left(\tau^{\prime}\right) d \tau^{\prime}+\xi(\tau)
$$

Assuming that $\gamma$ has been determined in advance by (3.10), $\rho$ can be obtained by solving (3.18), the result substituted in (3.17) and the integration performed to obtain $\sigma^{2}$.

The derivation given here is valid under an irreducibility assumption which is satisfied for our model except at the values $f=0$ and $f=1$ of the flowering fraction. The corresponding values of $\sigma^{2}=\sigma^{2}(f)$ are easily found by direct computation,

$$
\begin{aligned}
& \sigma^{2}(0)=0, \\
& \sigma^{2}(1)=\frac{1}{2} \psi^{\prime}(a),
\end{aligned}
$$

where $\psi^{\prime}(a)$ denotes the trigamma function. Since the formula (3.19b) was stated without proof in I and will be important below we give a short derivation in Appendix B.

\section{Numerical calculations}

In this section we calculate the variance of the geometric growth rate for the same parameter values as we did in I. To do this we solve the integral equation 
(3.18) and perform the quadrature (3.17) for a number of values of the flowering fraction $f$ by using routines from the NAG-library ${ }^{2}$.

We achieve good numerical stability except for values of the flowering fraction close to one. As $f$ approaches unity the parameter $z$ defined in (3.7) goes to zero and the algorithm becomes unstable. This is the reason that the data point for $f=0.95$ is missing in Fig. 1b. One could perform a small- $z$ expansion (similar to I, Sect. 5) in order to cover the region near $f=1$, but we will not do this here.

As in I we fix $s$ at the value 0.9 and consider the following cases
(a) $\bar{\phi}=2, \bar{\phi}=1$;
(b) $\bar{\phi}=2, \overline{\bar{\phi}}=5$;
(c) $\bar{\phi}=3, \bar{\phi}=5$,

for the mean and variance of offspring number, which are defined by

$$
\bar{\phi}:=\mathbb{E}(\phi)=a / k, \quad \overline{\bar{\phi}}:=\operatorname{Var}(\phi)=a / k^{2},
$$
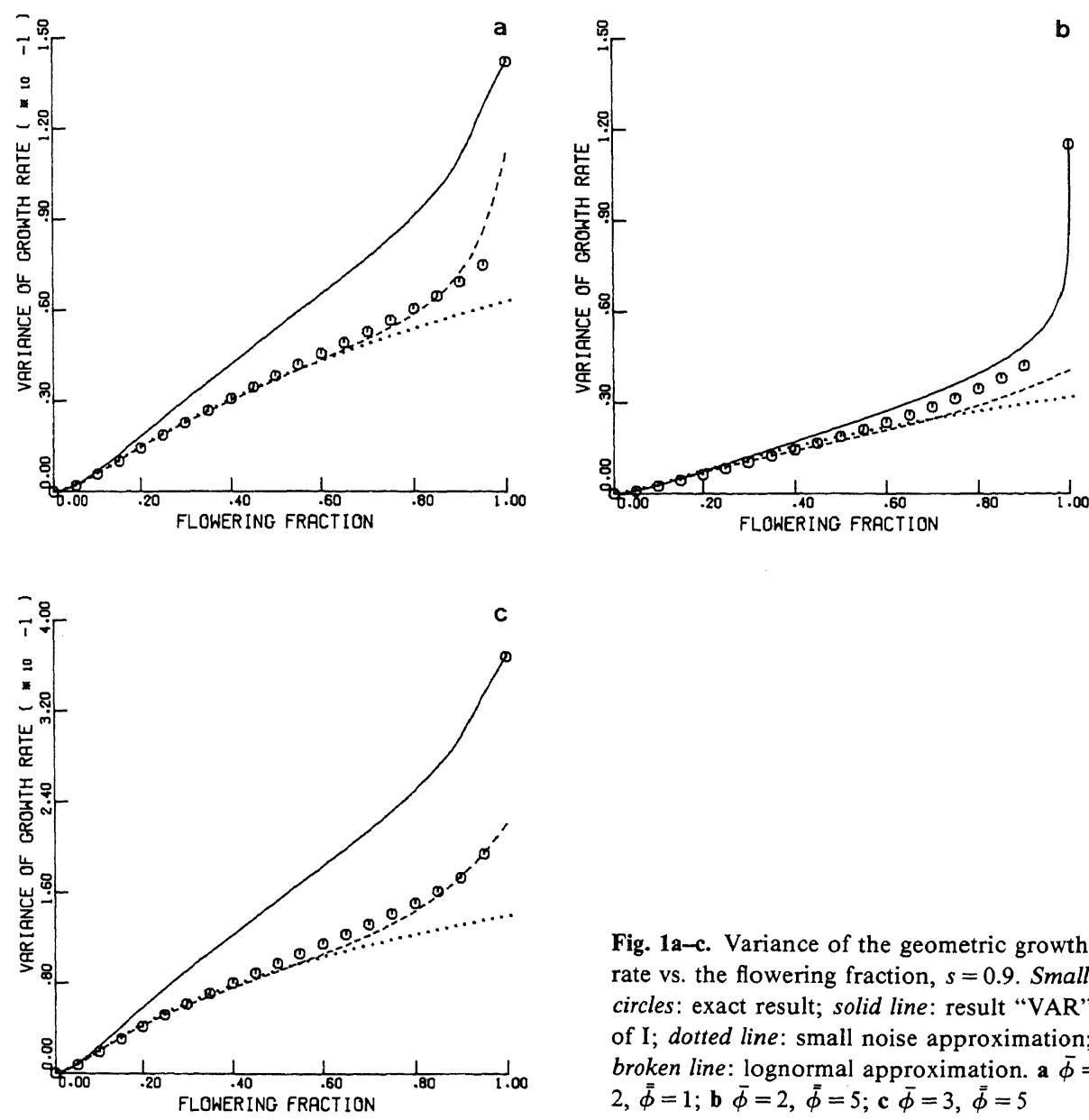

Fig. 1a-c. Variance of the geometric growth rate vs. the flowering fraction, $s=0.9$. Small circles: exact result; solid line: result "VAR" of I; dotted line: small noise approximation; broken line: lognormal approximation. a $\bar{\phi}=$ $2, \overline{\bar{\phi}}=1 ; \mathbf{b} \bar{\phi}=2, \bar{\phi}=5 ; \mathbf{c} \bar{\phi}=3, \bar{\phi}=5$

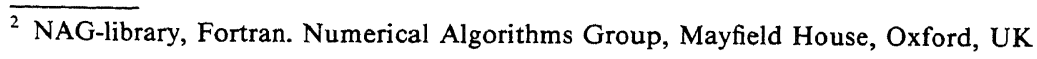


where $a$ and $k$ are the parameters of the gamma distribution (1.2). The results are shown as the small circles in Fig. 1. The solid curves represent the contribution "VAR" of I and are included to facilitate the comparison with the exact results. Also included are the small noise and lognormal approximations as discussed in I.

One sees that the steep decrease of $\sigma^{2}$ near $f=1$ is more pronounced than in the approximation "VAR" of I but the qualitative behaviour is the same. Also the small noise and lognormal approximations (the latter in particular) are much more accurate than appeared from Fig. 3 of I. Nevertheless, the discrepancy between the exact result and these approximations as $f$ approaches unity is unaffected, since the correct value (3.19b) at $f=1$ was used in I. The curves for the extinction probability (Fig. 6 of I) hardly change if the exact value of $\sigma^{2}$ is used and therefore we do not include them here.

\section{Appendix A}

Here we indicate how the results (3.17) and (3.18) can be obtained. From (3.11) we have

$$
\ln |X \bar{n}|=\ln \left\{1+(1-f) \tau^{\prime}\right\}+\ln \{s(1-f)(1+\tau)\}-\ln \{1+(1-f) \tau\}:=a\left(\tau, \tau^{\prime}\right),
$$

where $\tau^{\prime}$ is the first iterate of $\tau$,

$$
\tau^{\prime}=\eta \phi /(1+\tau)
$$

with $\eta$ given by (3.4). Writing (1.8) and (2.17) in terms of the variables $\tau$ and $\tau^{\prime}$ one has

$$
\mathrm{VAR}=\iint\left[a\left(\tau, \tau^{\prime}\right)\right]^{2} K\left(\tau^{\prime}, \tau\right) d \tau^{\prime} d \nu(\tau)-\gamma^{2}
$$

and

$$
\operatorname{COVAR}=2 \iint a\left(\tau, \tau^{\prime}\right) f_{1}\left(\tau^{\prime}\right) K\left(\tau^{\prime}, \tau\right) d \tau^{\prime} d \nu(\tau)
$$

where $d \nu(\tau):=h(\tau) d \tau$, with $h(\tau)$ the invariant density (3.6). Making the same variable change in (3.13) the expression for $\varepsilon(\tau)$ becomes

$$
\varepsilon(\tau)=\xi(\tau)-\ln \{1+(1-f) \tau\}+\int K\left(\tau^{\prime}, \tau\right) \ln \left\{1+(1-f) \tau^{\prime}\right\} d \tau^{\prime},
$$

where $\xi(\tau)$ is defined in (3.16).

Inserting (A1) in (A3)-(A4) and using $(3.9 \mathrm{~b}, \mathrm{c})$ and $(3.14 \mathrm{a})$ we find

$$
\begin{aligned}
\operatorname{VAR}= & \int\left[2 \alpha^{2}(\tau)+\beta^{2}(\tau)-2 \alpha(\tau) \beta(\tau)\right] d \nu(\tau) \\
& +\iint 2[\beta(\tau)-\alpha(\tau)] \alpha\left(\tau^{\prime}\right) K\left(\tau^{\prime}, \tau\right) d \tau^{\prime} d \nu(\tau)-\gamma^{2}
\end{aligned}
$$

and

$$
\operatorname{COVAR}=2 \int\{\alpha(\tau)-\beta(\tau)\} \varepsilon(\tau) d \nu(\tau)+2 \int \beta(\tau) f_{1}(\tau) d \nu(\tau)
$$

where

$$
\alpha(\tau)=\ln \{1+(1-f) \tau\}, \quad \beta(\tau)=\ln \{s(1-f)(1+\tau)\}
$$


Now insert equation (A5) for $\varepsilon(\tau)$ in (A7) and express $f_{1}(\tau)$ in terms of the function $\rho(\tau)$ as defined in (3.15). Then addition of the result to (A6) followed by a cancellation of various terms and the use of (3.10), (3.14b) leads to (3.17).

Finally, from (3.14), (3.15) and (A5) the following integral equation for $\rho$ is obtained,

$$
\rho(\tau)=\xi(\tau)+\int K\left(\tau^{\prime}, \tau\right) \rho\left(\tau^{\prime}\right) d \tau^{\prime},
$$

with

$$
\int \rho(\tau) h(\tau) d \tau=\int \ln \{1+(1-f) \tau\} h(\tau) d \tau .
$$

The solution of (A9a) is determined up to an additive constant which is fixed by (A9b). Since from (3.10) and (3.16).

$$
\int \xi(\tau) h(\tau) d \tau=0
$$

it follows from (3.17) that an additive constant in $\rho$ does not contribute to the variance $\sigma^{2}$. So instead of (A9b) we may as well use the normalization condition

$$
\int \rho(\tau) h(\tau) d \tau=0
$$

Subtracting (A11) from (A9a) one finally obtains Eq. (3.18), the solution of which is unique and automatically satisfies the normalization condition (A11).

\section{Appendix B}

We give here the derivation of Eq. (3.19b) for $\sigma^{2}(1)$. For $f=1$ Eq. (1.1) leads to

$$
N_{1,2 t}=\left\{s^{t} \prod_{i=1}^{t} \phi_{2 i-1}\right\} N_{1,0} ; \quad N_{2,2 t}=\left\{s^{t} \prod_{i=1}^{t} \phi_{2 i}\right\} N_{2,0},
$$

where we take the time parameter to be even. In the following we only look at the growth rate of $N_{1}$; for $N_{2}$ the same result is obtained. From (B1) we have

$$
\ln \left\{\frac{N_{1,2 t}}{N_{1,0}}\right\}=t \ln s+\sum_{i=1}^{t} \ln \left(\phi_{2 i-1}\right) .
$$

Hence, from the definitions (1.5) and (1.7), we find

$$
\gamma=\frac{1}{2}[\ln s+\mathbb{E}\{\ln \phi\}]
$$

and

$$
\sigma^{2}=\frac{1}{2}\left[\mathbb{E}\left\{(\ln \phi)^{2}\right\}-(\mathbb{E}\{\ln \phi\})^{2}\right]
$$

Define

$$
\Delta(a, k):=\int_{0}^{\infty} \phi^{a-1} e^{-k \phi} d \phi=k^{-a} \Gamma(a),
$$

where $\Gamma(a)$ is the gamma function. Then

$$
\mathbb{E}\{\ln \phi\}=\frac{\partial / \partial a \Delta(a, k)}{\Delta(a, k)}=-\ln k+\psi(a),
$$

where

$$
\psi(a)=\Gamma^{\prime}(a) / \Gamma(a)
$$


and

$$
\mathbb{E}\left\{(\ln \phi)^{2}\right\}=\frac{\partial^{2} / \partial a^{2} \Delta(a, k)}{\Delta(a, k)}=(\ln k)^{2}-2(\ln k) \psi(a)+\frac{\Gamma^{\prime \prime}(a)}{\Gamma(a)} .
$$

Substituting (B6) and (B8) in (B4) we find

$$
\sigma^{2}=\frac{1}{2}\left\{\frac{\Gamma^{\prime \prime}(a)}{\Gamma(a)}-\left(\frac{\Gamma^{\prime}(a)}{\Gamma(a)}\right)^{2}\right\}=\frac{1}{2} \frac{d}{d a}\left(\frac{\Gamma^{\prime}(a)}{\Gamma(a)}\right)=\frac{1}{2} \psi^{\prime}(a),
$$

as was to be shown.

\section{References}

1. Roerdink, J. B. T. M.: The biennial life strategy in a random environment. J. Math. Biol. 26, 199-215 (1988)

2. Bougeerol, P., Lacroix, J.: Products of random matrices with applications to Schrödinger operators (Progress in probability and statistics, vol. 8) Boston: Birkhäuser 1985

Received October 3, 1988/Revised January 13, 1989 